\title{
Acupressure has not an analgesic effect in patients with renal colic: a randomized controlled trial
}

\author{
Ehsan Bolvardi*, Bahram Zarmehri*, Seyed Mojtaba Mousavi Bazzaz**, \\ Ali Khorsand Vakilzadeh****, Mahdi Foroughian*, Hasan Hoseini Faegh ${ }^{\dagger}$, \\ and Neema John Mehramiz
}

\begin{abstract}
\section{BACKGROUND}

Easing renal colic (RC) pain is of the most important issues in the emergency departments. This study was conducted to investigate the analgesic effects of intravenous paracetamol in comparison to acupressure and intramuscular diclofenac in patients with RC.

\section{METHODS}

This randomized clinical trial was conducted on 96 eligible patients with RC, visiting the Emergency Department of Imam Reza Hospital of Mashhad, Iran. The participants were selected by consecutive non-random sampling method, and were randomized in three groups, each comprising of 32 participants. The first and second groups received $75 \mathrm{mg}$ intramuscular diclofenac and $1 \mathrm{~g}$ intravenous paracetamol, respectively. In the third group, three acupressure points were manually stimulated for six minutes. The pain score was measured by Visual Analog Scale for pain at baseline and after 10, 30, and 60 minutes. Collected information analyses by SPSS version 20 and interpreted using descriptive statistics (frequency, percentage) and inferential statistics (chi-square test).
\end{abstract}

\section{RESULTS}

The mean age was $31.60 \pm 7.42$ years (range: $18-50$ ). The two methods (diclofenac and paracetamol groups) were significantly better in pain reduction compared to acupressure after 10 minutes $(p<0.001)$. Ninety four percent of participants in diclofenac group, $87 \%$ of paracetamol group and none of acupressure group were completely satisfied with their treatment $\operatorname{method}(\mathrm{p}<0.001)$.

\section{CONCLUSION}

This study demonstrated that acupressure did not effectively reduces renal colic pain. It seems that contrary to acupressure, intravenous paracetamol is a safe and effective analgesic medicine for patients with RC.

Keywords: Renal colic, satisfaction, paracetamol, acupressure, diclofenac

Cite this article as: Bolvardi E, Zarmehri B, Bazzaz MM, et al. Acupressure has not an analgesic effect in patients with renal colic: a randomized controlled trial. Univ Med 2019;38:10-6. doi: 10.18051/UnivMed.2019.v38.10-16

\author{
*Department of Emergency Medicine, \\ Faculty of Medicine, Mashhad \\ University of Medical sciences, \\ Mashhad, Iran \\ **Department of Community \\ Medicine, Faculty of Medicine, \\ Mashhad University of Medical \\ Sciences, Mashhad, Iran \\ ***School of Persian and \\ Complementary Medicine, Faculty of \\ Medicine, Mashhad University of \\ Medical Sciences, Mashhad, Iran \\ ${ }^{\dagger}$ Resident of Emergency Medicine, \\ Emergency Medicine Department, \\ Faculty of Medicine, Mashhad \\ University of Medical sciences, \\ Mashhad, Iran \\ *University of Arizona, College of Medicine, \\ Tucson, AZ, USA
}

\section{Correspondence:}

Mahdi Foroughian

Department of Emergency Medicine, Faculty of Medicine, Mashhad University of Medical sciences, Imam Reza Hospital, Imam Reza square, Mashhad, Iran

Postal code: 9137913316

Phone: 05138525312;09151240686

Email: foroughianmh@mums.ac.ir ORCID ID : 0000-0002-3944-9361

Date of first submission, September 27, 2018

Date of final revised submission,

February 9, 2019

Date of acceptance, February 11, 2019

This open access article is distributed under a Creative Commons AttributionNon Commercial-Share Alike 4.0 International License 


\section{INTRODUCTION}

Pain is a physiological response to a harmful stimulation. The expression of pain depends on many factors including emotional, physical, and cultural states of an individual. ${ }^{(1)}$ As pain presents $75-80 \%$ of emergency primary compliant, proper pain management is an important task of physicians in the emergency department (ED. ${ }^{(1)}$

Patients with renal colic (RC) comes to ED with severe pain in flank or abdomen which needs immediate treatment. ${ }^{(2)}$ The urinary system consists of the kidneys, ureters, bladder, and the urethra (urinary meatus). Renal colic pain is the most common urinary system disorder. ${ }^{(3)}$ Renal colic is associated with severe pain, which begins from flanks and spreads to the groin. It is a recurring pain with the chance of exacerbation. The standard RC treatment includes relieving pain and correcting water and electrolyte imbalance. The only definite treatment is the elimination of the pain source, i.e., the cause of obstruction. However, the majority of initial treatments at admission are bound to temporary pain relief. ${ }^{(1,4)}$ Morphine and some other opiate compounds are the most effective analgesics; however, some concerns regarding their complications, such as physical dependence, substance abuse, inhibition of respiratory center, and sensitivity reactions, have signified the need for alternative methods. As a result, there is currently a greater tendency towards the use of nonsteroidal antiinflammatory drugs (NSAIDs). ${ }^{(5-8)}$ On the other hand, NSAIDs have complications as gastrointestinal bleeding and renal failure. ${ }^{(9)}$ According to aforementioned complications, it is necessary to use less complicated and more effective medications for RC.

The efficacy of intravenous paracetamol for pain control in therapeutic dose (1 gr four times a day in adult patients) can be compared with morphine or ketorolac, and because of fewer side effects it can be a treatment option in renal colic for patients presenting to ED. ${ }^{(10)}$
Regarding the abundant problems and complications of pharmacotherapy, the use of non-medical methods that results in pain reduction in $\mathrm{RC}$ patients seems logical. In recent years, the application of non-medical methods, known as alternative medicine, has been given special attention. Acupressure is a non-medicinal, non-aggressive, and relatively inexpensive method, which has almost no side effect. It is an effective technique for pain reduction without any complication. The acupressure helps to rejuvenate the body merely through applying a pressure on a certain body points by hands. It is a derivative of the Chinese medicine. ${ }^{(11-13)}$ In other words, acupressure is a type of massage that stimulates certain body points to increase circulation and reduce muscle tension. Acupressure is a means of stimulation of the same acupoints as used in acupuncture but without the use of needles or other medical instruments. ${ }^{(14)}$ Some studies have reported positive effects of pain reduction for some medical conditions like dysmenorrhea, neck pain, and fractures. ${ }^{(15-17)}$ However, to the best of our knowledge, no study has focused on effectiveness of acupressure on pain reduction in RC.

In addition to the financial burden, the use of medicines, such as opiates and NSAIDs, is associated with many side effects including hypoventilation, nausea, purpura, and bleeding. ${ }^{(18)}$ According to many complications from nonsteroidal and morphine medications, it is necessary to use less complicated and more effective RC medications. So, we intended to compare the analgesic effects of intravenous paracetamol, acupressure, and intramuscular diclofenac in patients with RC.

\section{METHODS}

\section{Research design}

This randomized clinical trial was performed at the emergency departments of Imam Reza Hospital in Mashhad, Iran. This study performed from January to June 2017. 


\section{Research subjects}

The participants were selected from 1850 years old patients visiting the Emergency Department (ED) of Imam Reza Hospital of Mashhad, Iran. Inclusion criteria were patient coming to ED with RC, accepting the study and completing the informed consent form. Exclusion criteria were the history of drug dependence, patients with allergy to medications of the present study, pregnant women, and patients who had used other analgesics before admission. Also, patients who developed any unwanted medical complication during the study were excluded.

The sample size was calculated using the formula for comparison of mean between two independent samples considering these parameters: alpha error $=0.05$, beta error $=0.2$, $\mathrm{x}_{1}=9.1, \mathrm{SD}_{1}=2.1, \mathrm{x}_{2}=8.5, \mathrm{SD}_{2}=2.1, \mathrm{x}_{3}=5.1$, $\mathrm{SD}_{3}=1.2$. The optimal sample was 32 for each group. ${ }^{(19)}$

\section{Intervention}

The patients were randomized into three equal-sized groups using a random number sequence and were matched regarding gender. The randomization scheme was generated by using the Web site Randomization.com (http:// www.randomization.com).

The first and second group received $75 \mathrm{mg}$ intramuscular diclofenac and $1 \mathrm{~g}$ intravenous paracetamol, respectively. In the third group, three acupoints (first web of fingers, the second web of toes, and a point on the medial side of distal tibia, above the medial malleolus) were selected according to the Chinese medicine. These points were stimulated under a certain amount of pressure (4-5kg) every 2-3 seconds for 5 seconds. This process was performed for an overall time of six minutes. The researcher who performed the acupressure (AKV) had a certified training in clinical acupressure by China Beijing International Acupuncture Training Center) CBIATC (, and have experiences in acupressure for more than 10 years. It is worth noting that an alternative method (morphine therapy) was used if the patients did not respond desirably to pain 15 minutes after the intervention.

The pain level of all groups was measured after 10, 30, and 60 minutes between the three groups. Pain score was categorized into three levels: severe pain (score: 7-10), moderate pain (score: 4-6) and mild pain (score: 0-3). Besides, the level of patient satisfaction (not satisfied, moderately satisfied, and completely satisfied) and possible side effects in each method were also recorded.

\section{Pain measurements}

The demographic information including gender, age, and weight were obtained. The research colleague, who was blinded to the patients' groups, measured the pain level of all patients, using Visual Analog Scale at baseline and after the intervention.

\section{Statistical analysis}

Then, the collected information was analyses by SPSS version 20 and interpreted using descriptive statistics (frequency, percentage) and inferential statistics (chi-square test). The significance level was considered below 0.05 .

\section{Ethical clearance}

First, the advantages and disadvantages of all three study methods were explained to the participants, and their informed written consent was collected. This study was approved by Mashhad University of Medical Sciences (940520) and Ethical Committee of Mashhad University of Medical Sciences (IR.MUMS.FM.REC.1395.79). This study was also registered at Iranian Registry for Clinical Trials (IRCT2016072129023N1).

\section{RESULTS}

In this study 134 patients assessed for eligibility. Thirty-eight patients excluded from the study (Not meeting inclusion criteria $=26$ patients, declined to participate $=12$ patients). Finally, we randomized 96 patients with RC 


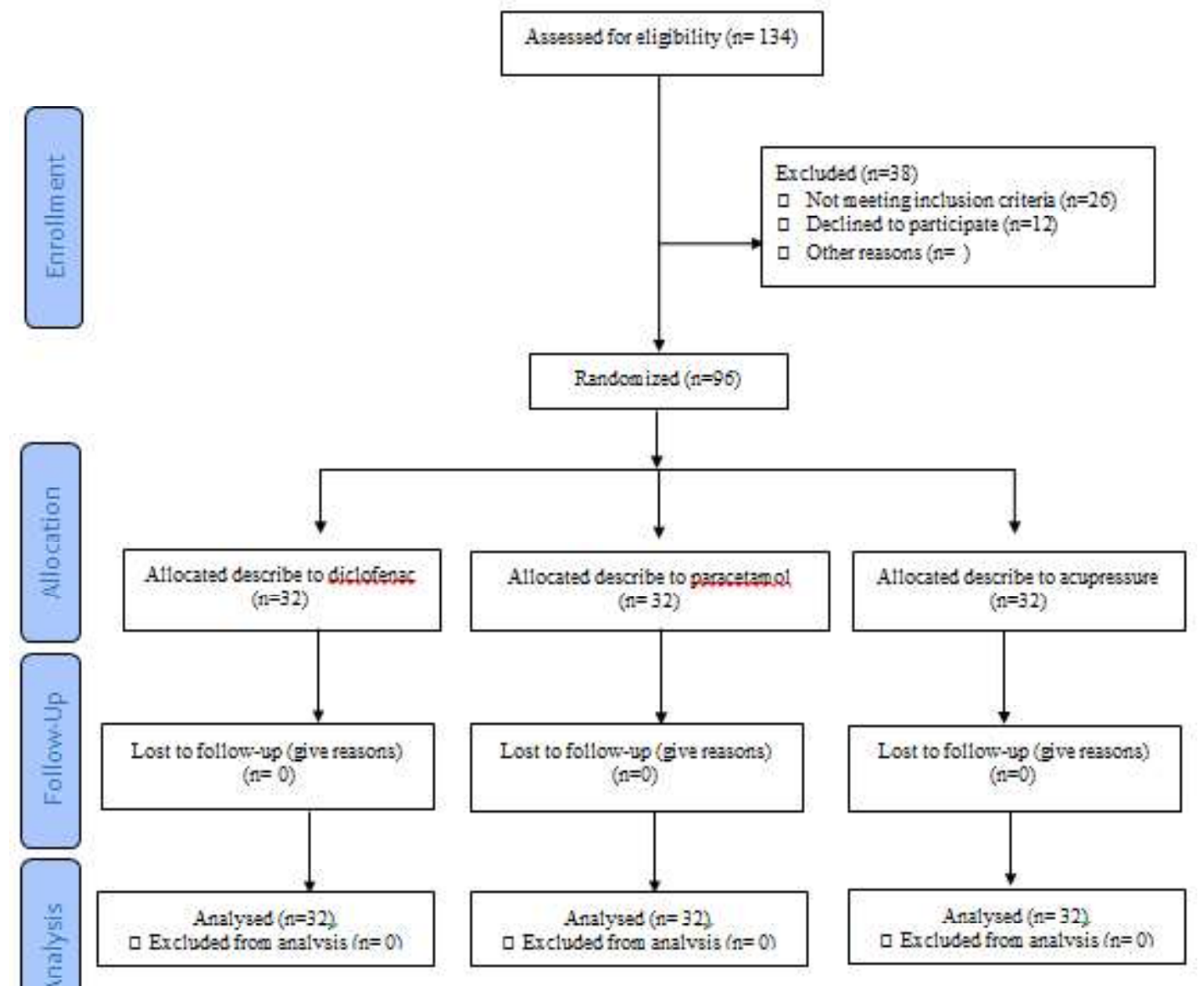

Figure 1. Flow diagram of the participants

visiting the ED to three groups of intervention (Figure 1). The mean age was $31.60 \pm 7.42$ years (range: 18-50). Half of admitted patients were between 27-34 years old (50\%). Most patients were male $(84.4 \%, 81$ individuals). Concerning BMI, $55.2 \%$ of patients had normal weight. Table 1 shows that there was no statistical difference between groups regarding basic characteristics and baseline pain. This meciss that the randomization was successfully distributed the other variable between the three groups.

As the acupressure group have severe pain after 10 min morphine therapy substituted and for this reason, we did not compare it to other groups that have pain relief in 30 and 60 minutes. As Table 2 shows, $96.9 \%$ of participants in acupressure group still had severe pain after 10 minutes, this was $6.2 \%$ for diclofenac group and $9.4 \%$ for paracetamol group $(\mathrm{p}<0.001)$. It is should be reminded that due to the lack of pain reduction in acupressure group after 15 minutes, the morphine therapy was substituted for the acupressure. No side effects were observed for any participants.

Finally, while $93 \%$ (30) of participants in diclofenac group and $87.5 \%$ (28) of participants in paracetamol group were completely satisfied, 96.9\% (31) of participants in acupressure group were not satisfied with their treatment method for pain reduction $(\mathrm{p}<0.001)$ (Table 2$)$.

\section{DISCUSSION}

This study showed no significant difference in pain reduction between the paracetamol and diclofenac groups after 10, 30, and 60 minutes, and the pain was relieved with passing time in both groups. In recent years, many studies have been conducted on the application of intravenous paracetamol. For example, Mofidi et al. ${ }^{(20)}$ 
Table 1. Base-line characteristics of the three intervention groups

\begin{tabular}{|c|c|c|c|c|}
\hline \multirow{2}{*}{ Characteristics } & \multicolumn{3}{|c|}{ Intervention } & \multirow{2}{*}{ p value } \\
\hline & Diclofenac $(n=32)$ & Paracetamol $(n=32)$ & Acupressure $(n=32)$ & \\
\hline \multicolumn{5}{|l|}{ Gender } \\
\hline Male & $27(84.4)$ & $28(87.5)$ & $26(81.2)$ & 0.789 \\
\hline Female & $5(15.6)$ & $4(12.5)$ & $6(18.8)$ & \\
\hline \multicolumn{5}{|l|}{ Age (years) } \\
\hline $18-26$ & $4(12.5)$ & $6(18.8)$ & $3(9.4)$ & 0.860 \\
\hline $27-34$ & $18(56.2)$ & $14(43.8)$ & $16(50.0)$ & \\
\hline $35-42$ & $8(25.0)$ & $11(34.3)$ & $11(34.3)$ & \\
\hline $43-50$ & $2(6.3)$ & $1(3.1)$ & $2(6.3)$ & \\
\hline \multicolumn{5}{|l|}{ BMI $\left(\mathrm{kg} / \mathrm{m}^{2}\right)$} \\
\hline Underweight & $4(12.5)$ & $5(15.6)$ & $3(9.4)$ & 0.217 \\
\hline Normal & $17(53.1)$ & $14(43.8)$ & $22(68.8)$ & \\
\hline Overweight & $11(34.4)$ & $11(34.4)$ & $5(15.6)$ & \\
\hline Obese & $0(0.0)$ & $2(6.2)$ & $2(6.2)$ & \\
\hline \multicolumn{5}{|l|}{ Pain } \\
\hline Severe & $28(87.5)$ & $27(84.4)$ & $26(81.2)$ & 0.708 \\
\hline Moderate & $3(9.4)$ & $4(12.5)$ & $6(18.8)$ & \\
\hline Mild & $1(3.1)$ & $1(3.1)$ & $0(0.0)$ & \\
\hline
\end{tabular}

Data presented as n (\%); BMI : body mass index

compared the effectiveness of intravenous paracetamol and tramadol for RC patients. Their findings suggested the greater effectiveness of the former medication in relieving pain in renal patients. Serinken et al. ${ }^{(6)}$ and Bektas et al. ${ }^{(7)}$ compared the effects of paracetamol and morphine in RC patients and did not find any significant difference. In Iran, Baghi et al. ${ }^{(21)}$ compared the effectiveness of low-dose and highdose intravenous paracetamol-morphine combinations in treating RC patients. Results did not show any significant difference in pain reduction and side effects. Van Aken et al. ${ }^{(22)}$ in a study compared the effectiveness of a singledose intravenous paracetamol-intramuscular morphine and its repetition in improving analgesia after dental surgery. They did not find any specific statistical and clinical difference between the paracetamol and morphine groups regarding pain reduction; however, there were fewer side effects in the paracetamol group.

Comparison of our findings with results from other studies showed that paracetamol is a safer and more effective medication in pain reduction for patients with renal pain. This medication can produce desirable analgesic effects without significant complications. Regarding the lack of access to intravenous NSAIDs in some medical centers and many complications associated with opiates (nausea, vomiting, seizure, etc.), the intravenous paracetamol can used as a suitable substitute for pain reduction in RC patients.

Table 2. Comparison of analgesic effects and satisfaction score between the intervention groups after 10 minutes

\begin{tabular}{lcccc}
\hline \multirow{2}{*}{ Outcome criteria } & \multicolumn{3}{c}{ Intervention } & \multirow{2}{*}{ p value } \\
\cline { 2 - 4 } & Diclofenac (n=32) & Paracetamol (n=32) & Acupressure (n=32) & \\
\hline Pain after 10 minutes & $2(6.2)$ & $3(9.4)$ & $31(96.9)$ & $<0.001$ \\
Severe & $16(50.0)$ & $22(68.8)$ & $1(3.1)$ & \\
Moderate & $14(43.8)$ & $7(21.9)$ & $0(0.0)$ & \\
Mild & & & & \\
Satisfaction & $30(93.8)$ & $28(87.5)$ & $1(0.0)$ & 0.001 \\
Completely & $2(6.2)$ & $4(12.5)$ & $31(96.9)$ & \\
Moderately & $0(0.0)$ & $0(0.0)$ & & \\
Not satisfied & & &
\end{tabular}

Data presented as n (\%) 
Results from the current study showed a significant difference in the pain score between the diclofenac and acupressure groups, and also between the paracetamol and acupressure group 10-minute after the interventions. Previous studies which were conducted on the effectiveness of acupressure have reported controversial findings. For example, there are studies about the effectiveness of acupressure on dysmenorrhea, ${ }^{(15)}$ neck pain, ${ }^{(16)}$ pain after distal radial fractures, ${ }^{(17)}$ and pain caused by bone marrow aspiration in cancer patients. ${ }^{(23)}$ Results of these studies do not comply with our findings. This contradiction can be attributed to the different effect of acupressure on different types and levels of pain: regarding the high-intensity level of RC pain, acupressure may not have a considerable effect on RC pain.

This study was not without limitations. First, we used a subjective tool for measuring pain. Second, personal characteristics and even belief to modern or alternative medicine can influence the perception of pain. Third, the natural difference between the acupressure therapy and the two pharmacological therapies did not allow us to perform a participant blinding. However, designing an RCT for comparison three treatment methods in one of the most painful conditions in medicine is one of the strengths of this study. Future studies with patients in other painful conditions can improve the results.

\section{CONCLUSION}

Acupressure was not found to be an effective analgesic in RC. Regarding the side effects of opioid analgesics and NSAIDs, it seems that intravenous paracetamol can be considered as an effective and safe pain medication in emergencies.

\section{ACKNOWLEDGEMENTS}

This study was financially supported by Mashhad University of Medical Sciences and we thank our patients for their cooperation.

\section{CONFLICT OF INTEREST}

None to be declared.

\section{CONTRIBUTORS}

Study design, data gathering and analysis performed by EB, BZ, SM-MB, AKV, MF, HHF, NJM. Acupressure done by AKV. Manuscript drafting done by all authors, and all authors accepted the final version of manuscript.

\section{REFERENCES}

1. Tintinalli JE, Stapczynski JS, Ma OJ, et al. Tintinalli's emergency medicine : a comprehensive guide. $8^{\text {th }}$ ed. New York: Mc Graw Hill; 2016.

2. Soylu A, Sarier M, Altunoluk B, et al. Comparison of the efficacy of intravenous and intramuscular lornoxicam for the initial treatment of acute renal colic: a randomized clinical trial .Urol J 2018;1:1-6. doi: 10.22037/uj.v0i0.4496.

3. Bultitude M, Rees J. Management of renal colic. BMJ 2012;345:e5499. doi: https://doi.org/10.1136/ bmj.e5499.

4. Frassetto L, Kohlstadt I. Treatment and prevention of kidney stones: an update. Am Fam Physician 2011;84:1234-42.

5. Talebi Doluee M, Reihani H, Zarmehri B, et al. Pain management in emergency department: intravenous morphine vs. intravenous acetaminophen. Rev Clin Med 2015;2:103-6. doi: 10.17463/RCM.2015.02.012.

6. Serinken M, Eken C, Turkcuer I, et al. Intravenous paracetamol versus morphine for renal colic in the emergency department: a randomised doubleblind controlled trial. Emerg Med J 2012;29:902-5. doi: 10.1136/emermed-2011-200165.

7. Bektas F, Eken C, Karadeniz O, et al. Intravenous paracetamol or morphine for the treatment of renal colic: a randomized, placebo-controlled trial. Ann Emerg Med 2009;54:568-74. doi: 10.1016/ j.annemergmed.2009.06.501.

8. Talebi Deloee M, Zarmehri B, Pishbin E, et al. Intravenous acetaminophen versus intravenous morphine sulfate for isolated diaphyseal long bone fractures: a randomized clinical trial. Trauma Monthly 2017;22:e57680. doi: 10.5812/ traumamon.57680.

9. Davis A, Robson J. Editorial: the dangers of NSAIDs: look both ways. Br J Gen Pract 2016;66:172-3. doi: 10.3399/bjgp16X684433. 
10. Morgan S. Intravenous paracetamol in patients with renal colic. Emerg Nurse 2011;18:22-5. doi: 10.7748/en2011.02.18.9.22.c8337.

11. Lee YH, Eom KM, Seo HS, et al. Effects of acupressure on vertebra artery blood flow in tension-type headache patients. Korean J Acupunc 2011;28:49-58. doi: 10.1016/ j.jams.2011.10.005.

12. Najafi F, Jaafarpour M, Sayehmiri K, et al. An evaluation of acupressure on the Sanyinjiao (SP6) and Hugo (LI4) points on the pain severity and length of labor: a systematic review and netaanalysis study. Iran J Nurs Midwifery Res 2018;23:1-7.doi: 10.4103/ijnmr.IJNMR_184_15.

13. Kao MJ, Hsieh YL, Kuo FJ, et al. Electrophysiological assessment of acupuncture points. Am J Phys Med Rehabil 2006;85:443-8. doi: 10.1097/01.phm.0000214308.02631.9f.

14. Lin GH, Chang WC, Chen KJ, et al. Effectiveness of acupressure on the Taichong acupoint in lowering blood pressure in patients with hypertension: a randomized clinical trial. Evid Based Complement Alternat Med 2016; Article ID 1549658, 9 pages. doi: 10.1155/2016/1549658.

15. Mirbagher-Ajorpaz N, Adib-Hajbaghery M, Mosaebi F. The effects of acupressure on primary dysmenorrhea: a randomized controlled trial. Complement Ther Clin Pract 2011;17:33-6. doi: 10.1016/j.ctcp.2010.06.005.

16. Lee EJ, Frazier SK. The efficacy of acupressure for symptom management: a systematic review. J Pain Symptom Manage 2011;42:589-603. doi: 10.1016/j.jpainsymman.2011.01.007.
17. Lang T, Hager H, Funovits V, et al. Prehospital analgesia with acupressure at the Baihui and Hegu points in patients with radial fractures: a prospective, randomized, double-blind trial. Am J Emerg Med 2007;25:887-93. doi: 10.1016/ j.ajem.2007.01.016.

18. Kennedy D. Analgesics and pain relief in pregnancy and breastfeeding. Aust Prescr 2011;34:8-10.

19. Kaynar M, Koyuncu F, Buldu I, et al. Comparison of the efficacy of diclofenac, acupuncture, and acetaminophen in the treatment of renal colic. Am J Emerg Med 2015;33:749-53. doi: 10.1016/ j.ajem.2015.02.033.

20. Mofidi M, Shaker H, Ghafouri H. Comparison of analgesia effect between intravenous propacetamol and tramadol. J Mazandaran Univ Med Sci 2007;17:81-7.

21. Morteza-Bagi HR, Amjadi M, Mirzaii-Sousefidi R. The comparison of apotel plus low dose of morphine and full dose of morphine in pain relief in patients with acute renal colic. Addict Health 2015;7:66-73.

22. Van Aken H, Thys L, Veekman L, Buerkle H. Assessing analgesia in single and repeated administrations of propacetamol for postoperative pain: comparison with morphine after dental surgery. Anesth Analg 2004;98:15965 .

23. Bao T, Ye X, Skinner, et al. The analgesic effect of magnetic acupressure in cancer patients undergoing bone marrow aspiration and biopsy: a randomized, blinded, controlled trial. J Pain Symptom Manage 2011;41:995-1002. doi: 10.1016/ j.jpainsymman.2010.08.012. 